\title{
Water Maser Emission and the Parsec-Scale Jet in NGC 3079
}

\author{
A. S. Trotter, J. M. Moran, \& L. J. Greenhill \\ Harvard-Smithsonian CfA, Cambridge, MA 02138, U.S.A.
}

\begin{abstract}
We have imaged the $\mathrm{H}_{2} \mathrm{O}$ maser and 22,8 , \& $5 \mathrm{GHz}$ continuum in the nucleus of NGC 3079, using the NRAO VLBA. The maser features are distributed over $\sim 2$ pc along an axis aligned with the plane of the $k p c-s c a l e ~ e d g e-o n$ molecular disk. The masers are not angularly coincident with any detected continuum emission. The two brightest continuum features, which trace a parsec-scale jet, have similar spectra that peak at frequencies $\nu>$ $5 \mathrm{GHz}$. We also detected faint maser emission along the jet axis.
\end{abstract}

\section{1. $\mathrm{H}_{2} \mathrm{O}$ Maser Emission in the Molecular Disk}

NGC 3079 is an edge-on spiral galaxy at a distance of $\sim 16 \mathrm{Mpc}$ that contains a luminous $\mathrm{H}_{2} \mathrm{O}$ maser in its nucleus (Henkel et al. 1984; Haschick \& Baan 1985). Figure 1a shows the parsec-scale structure of this maser from a VLBA observation in 1995 January. The bulk of the emission is in $\sim 0.01 \mathrm{pc}$ clumps, which are located in a $0.2-$ pc cluster containing the maser peak, at $957 \mathrm{~km} \mathrm{~s}^{-1}$ (Figure 1a, Inset). All these features are blueshifted with respect to the systemic velocity, $v_{\text {sys }}=1108 \pm 1 \mathrm{~km} \mathrm{~s}^{-1}$ (Irwin \& Seaquist 1991), and span a velocity range of $\sim 140 \mathrm{~km} \mathrm{~s}^{-1}$. Two additional blue clumps lie $\sim 0.5 \mathrm{pc}$ to the north. The only significantly redshifted emission is from a single feature at $v_{\mathrm{LSR}}=1190 \mathrm{~km} \mathrm{~s}^{-1}$ that lies $1.2 \mathrm{pc}$ to the south (Figure $1 \mathrm{~b}$ ). The maser distribution is aligned with the major axes of the kpc-scale stellar and CO disks (Sofue \& Irwin 1992), and the sense of $\Delta v$ is the same, which suggests common dynamics. However, the large velocity dispersion in the main cluster, as well as projected velocity gradients of $>10^{3} \mathrm{~km} \mathrm{~s}^{-1} \mathrm{pc}^{-1}$ within the clumps, indicate a non-rotational component of the maser velocity field.

\section{Evidence for a Nuclear Jet}

We observed two prominent compact continuum sources in the nucleus of NGC 3079 at frequencies $\nu=5,8$ and $22 \mathrm{GHz}$, which we identify with the $\nu=5 \mathrm{GHz}$ components A and B of Irwin \& Seaquist (1988). By phase referencing, we determined that component $B$ is offset 6.7 mas to the west of the maser peak, with an uncertainty of 0.1 mas. $A$ and $B$ are separated by $\sim 1.7 \mathrm{pc}$ along a position angle of $-55^{\circ}$. Both are resolved by our VLBA observations, suggesting that neither marks a compact central engine. A third compact $5 \mathrm{GHz}$ component $(\mathrm{X})$ was detected $\sim 4 \mathrm{pc} \mathrm{SE}$ of $\mathrm{B}$ along the same axis connecting $\mathrm{A}$ and $B$, which strongly indicates that the continuum traces a jet structure. The spectra of $A$ and $B$ are peaked, with spectral indices $\alpha \sim-1.6$ between 5 and $8 \mathrm{GHz}$ and $\alpha \sim 1$ between 8 and $22 \mathrm{GHz}\left(S \propto \nu^{-\alpha}\right)$. The structure and spectral properties are characteristic of the class of compact, symmetric $\mathrm{GHz}^{-}$-peaked spectrum (GPS) radio sources (e.g., O'Dea, Baum, \& Stanghellini 1991). Component $\mathrm{X}$ was not detected at $\nu>5 \mathrm{GHz}$, suggesting it has a steep spectrum. 

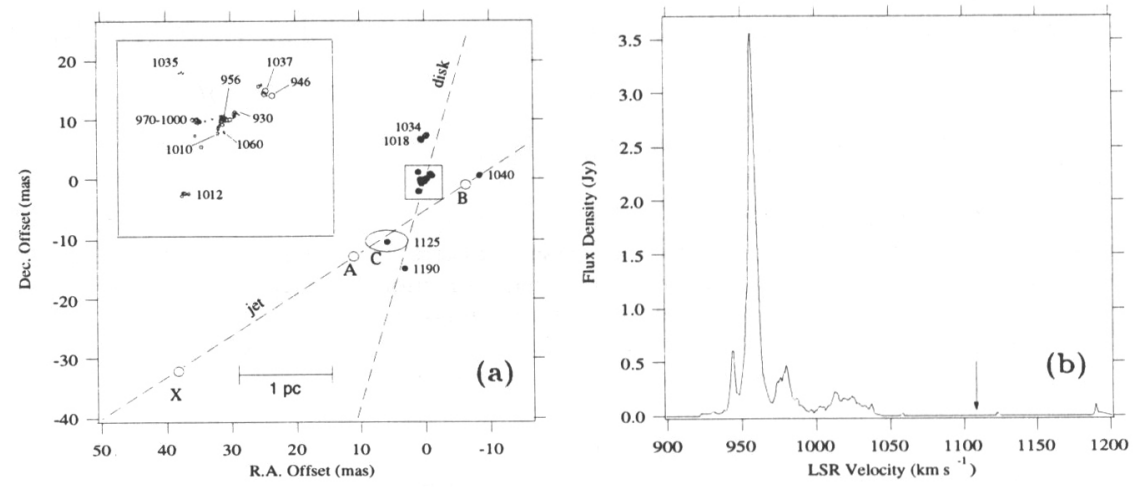

Figure 1. (a) Distribution of $\mathrm{H}_{2} \mathrm{O}$ maser and continuum emission in the nucleus of NGC 3079. Selected maser features are labeled with their LSR Doppler velocities (radio definition). Positions and designations of continuum jet features are indicated by $A, B, C a n d X$. Dashed lines trace the major axis of the kpc-scale molecular disk detected in $\mathrm{CO}$, and the axis of the pc-scale jet. Inset: Expanded view of the boxed region centered on the maser peak. (b) $\mathrm{H}_{2} \mathrm{O}$ maser spectrum. Total imaged flux density is plotted as a function of LSR velocity. The systemic velocity is indicated with an arrow.

\section{Relationship between Masers and Continuum}

By phase referencing, we determined that component $B$ has a projected offset $0.507 \pm 0.008 \mathrm{pc}$ to the west of the maser peak. None of the strong maser emission is coincident with detected continuum, which suggests that the maser does not amplify compact background sources. Lower-resolution $22 \mathrm{GHz}$ images show an extended continuum feature $(C)$ roughly midway between $A$ and $B$ that is absent from the 5 and $8 \mathrm{GHz}$ images. A maser feature at $1123 \mathrm{~km} \mathrm{~s}^{-1}$ is superimposed on this component. A second maser feature at $1040 \mathrm{~km} \mathrm{~s}^{-1}$ was detected $0.2 \mathrm{pc}$ to the NW of component $\mathrm{B}$. These two features may represent a second population of maser emission associated with the jet outflow. Ideal conditions for strong $\mathrm{H}_{2} \mathrm{O}$ maser emission can be generated downstream of molecular shocks (e.g., Elitzur, Hollenbach, \& McKee 1989). Such shocks could arise in supersonic turbulence in the inner molecular disk, or in bulk flows that are due to interaction of the jet outflow with the disk.

Acknowledgments. The National Radio Astronomy Observatory is a facility of the National Science Foundation, operated under a cooperative agreement by Associated Universities, Inc.

\section{References}

Elitzur, M., Hollenbach, D. J., \& McKee, C. F. 1989. ApJ, 346, 983-990.

Haschick, A. D., \& Baan, W. A. 1985. Nature, 314, 144-146.

Henkel, C., et al. 1984. IAUC, 3983, 1.

Irwin, J. A., \& Seaquist, E. R. 1988. ApJ, 335, 658-667.

Irwin, J. A., \& Seaquist, E. R. 1991. ApJ, 371, 111-130.

O'Dea, C. P., Baum, S. A., \& Stanghellini, C. 1991. ApJ, 380, 66-77.

Sofue, Y., \& Irwin, J. A. 1992. PASJ, 44, 353-365. 